\title{
Análisis de las complicaciones de la biopsia renal en el paciente trasplantado renal
}

\author{
David Berlango Martín*, María Dolores Navarro Cabello**, José Berlango Jiménez*, Pedro Aljama García**
}

*Enfermeros, ** Nefrólogos, UGC Nefrología. Hosp. U. Reina Sofía. Córdoba

\section{Resumen}

Antecedentes: La biopsia renal es una técnica fundamental en el estudio de la patología del injerto renal. Es fundamental conocer sus complicaciones ya que es el procedimiento más agresivo por su morbimortalidad.

Objetivos: El objetivo principal de nuestro estudio fue analizar e identificar las complicaciones clínicas derivadas de la biopsia renal percutánea en pacientes trasplantados renales. Evaluamos si algún factor clínico de los pacientes influye en la aparición de dichas complicaciones.

Pacientes y Métodos: Se realizó un estudio observacional, retrospectivo de todos los pacientes a los que se les realizó una biopsia renal percutánea de injerto renal entre enero de 2009 y enero 2012. Se analizaron: edad, sexo, hipertensión arterial, creatinina sérica, proteinuria y hemoglobina previa y posterior a la biopsia. Definimos complicaciones menores (caída hemoglobina mayor $1 \mathrm{~g} / \mathrm{dl}$, hematuria macroscópica) y complicaciones mayores (necesidad de transfusión, cirugía, nefrectomía, arteriografía, embolización o muerte). Todas las biopsias se realizaron con control ecográfico.

Correspondencia:

David Berlango Martín

Pje. José Manuel Rodríguez López Nº $101^{\circ} 2$ 14005 Córdoba

E-mail: david_berlango@hotmail.com
Resultados: El número de biopsias realizadas fue de 92. La edad media de los pacientes fue de $47.9 \pm$ 13.2 años y el $70.7 \%$ eran hombres. Se observaron 2 complicaciones mayores (2.2\%) y en ambas los pacientes presentaron sangrado y sepsis urológica, precisando uno de ellos de transfusión. Las complicaciones menores fueron del $14.1 \%$. No se encontró ninguna pérdida del injerto ni muerte por biopsia renal.

Conclusión: La biopsia percutánea en injerto renal realizada por médicos nefrólogos con experiencia y bajo control ecográfico en tiempo real, es un procedimiento con bajo riesgo, semejante al observado en riñón nativo. La cumplimentación cuidadosa de nuestro protocolo de biopsia renal, optimiza la relación riesgo beneficio de esta técnica.

\section{PALABRAS CLAVE:}

- BIOPSIA RENAL

- COMPLICACIONES

- INJERTO RENAL

\section{Analysis of renal biopsy complications in renal transplant patients}

\section{Abstract}

Background: Renal biopsy is a fundamental technique in the study of renal allograft pathology. It is essential to know its complications since it is the more aggressive procedure morbidity and mortality. 
Objectives: The main objective of our study is to analyze and identify clinical complications resulting from percutaneous renal biopsy in renal transplant patients. To assess whether a patient clinical factor influencing the onset of these complications.

Patients and Methods: An observational, retrospective study of all patients who underwent percutaneous renal biopsy renal graft between January 2009 and January 2012. Were analyzed: age, sex, hypertension, serum creatinine, proteinuria and hemoglobin before and after the biopsy. We defined minor complications (hemoglobin drop greater than $1 \mathrm{~g} / \mathrm{dl}$ ) and major complications (requiring transfusion, surgery, nephrectomy, arteriography, embolization or death). The biopsy was performed by the nephrology team with ultrasound guidance and withdrawing the antiplatelet therapy.

Results: The number of biopsies performed was 92. The mean age of patients was $47.9 \pm 13.2$ years and $70.7 \%$ were men. There were 2 major complications $(2.2 \%)$ and both patients had urological bleeding and sepsis, one need a transfusion. Minor complications were $14.1 \%$. The graft loss or patient death was not observed.

Conclusion: Percutaneous renal graft biopsy performed by physicians experienced nephrologists under ultrasound guidance in real time, is a low risk procedure, similar to that observed in native kidney. The careful completion of our protocol renal biopsy, optimize the risk benefit ratio of this technique.

\section{KEY WORDS:}

\section{- RENAL BIOPSY}

COMPLICATIONS

- RENAL GRAFT

\section{Introducción}

La biopsia renal percutánea (BRP) es una técnica muy útil para el diagnóstico de patologías renales, tanto en riñón nativo como en el injerto renal. El estudio histológico mediante biopsia percutánea del injerto renal, nos permite diferenciar de forma detallada la presencia de lesiones sugerentes de rechazo agudo o lesiones de tipo crónico. La técnica consiste en la extracción de un pequeño fragmento del tejido renal para su análisis histológico ${ }^{1}$. Todo ello con una elevada correlación clínico-patológica e internacionalmente aceptada $^{2,3}$.

Las primeras biopsias renales percutáneas mediante aguja y aspiración se llevaron a cabo en 1944 por Nils Alwall, pionero en la puesta en marcha de la hemodiálisis, aunque se le atribuyo el mérito a Antonio Pérez Ara ${ }^{4,5}$. Para la técnica utilizaban una aguja de Vim-Silverman modificada y colocaban al paciente en decúbito prono con una almohada bajo el abdomen ${ }^{4,5,6}$.

A pesar de todas las ventajas que tiene esta técnica existen algunas contraindicaciones absolutas como son el disponer de un único riñón (exceptuando el trasplante renal), padecer de hipertensión arterial no controlada, trastornos de la hemostasia, aneurismas en la arteria renal, el fallo de la biopsia percutánea con aguja, la obesidad mórbida y que el paciente no colabore ${ }^{4}$.

Esta técnica no está exenta de riesgos. Algunas de las complicaciones que pueden aparecer son la microhematuria, padecida por casi todos los pacientes; la hematuria macroscópica que aparece en un $12 \%$ de los casos, la hemorragia que es la complicación más grave (para su control es imprescindible que el paciente guarde reposo vigilando la tensión y el pulso, y supervisando la zona de punción y el color de la orina).

Para la detección de la hipotensión se hacen exhaustivos controles de constantes tras la biopsia. Otras complicaciones son el dolor severo en la zona de punción, requiriendo en muchos casos analgésicos para mitigar dicho dolor; fistula arteriovenosa (FAV), ocurre cuando la aguja de biopsia daña accidentalmente las paredes de una arteria y una vena cercanas, pudiéndose formar una conexión anormal entre los vasos; disminución de la hemoglobina que puede requerir de una transfusión ${ }^{7}$.

A pesar de todas estas posibles complicaciones, la biopsia renal percutánea es una técnica muy segura en la actualidad gracias a los avances técnicos en imagen, como la ecografía, que permiten la visualización de la aguja de biopsia y su trayectoria durante el procedimiento. Además, las agujas de biopsia han mejorado resultando sencillo su manejo al ser semiautomáticas ${ }^{8}$. 
La ecografía urgente es imprescindible ante la sospecha de complicaciones. En caso de hemorragia activa, el tratamiento con procedimientos endovasculares, con embolización selectiva del vaso sangrante, resuelve los problemas de forma eficaz. Alrededor de un $1 \%$ necesita transfusión, y un 0,1\% embolización o cirugía. El riesgo de mortalidad es extremadamente bajo en situaciones normales $<0^{\prime} 1 \% 9,10,11,12,13,14$. El paciente debe permanecer al menos 24 horas en el hospital, tras lo cual puede ser dado de alta si no han aparecido complicaciones.

A pesar de los importantes avances en el trasplante renal en cuanto a inmunosupresión, preservación del órgano y técnica quirúrgica, la supervivencia del injerto renal es de once años aproximadamente. Por tanto, parece necesario detectar la presencia de daño renal en el trasplantado cuando se está produciendo, antes del deterioro irreversible del injerto renal, para poder intervenir precozmente y así evitar el fallo del órgano y el inicio de nuevo de diálisis. Considerando que las complicaciones tras la biopsia son un factor muy importante a tener en cuenta, debemos intentar reducirlos en la medida de lo posible, puesto que la BRP es un procedimiento muy valioso y con una alta rentabilidad riesgo-beneficio.

La mayoría de estudios llevados a cabo sobre complicaciones tras biopsia percutánea son estudios realizados en las biopsias de riñones nativos, pero apenas existen estudios relativos a las complicaciones que pueden surgir al realizar una biopsia del injerto renal. Por este motivo nos planteamos como objetivo principal de nuestro estudio, analizar e identificar las complicaciones clínicas derivadas de la BRP en pacientes trasplantados renales y evaluar si algún factor clínico de los pacientes influyó en la aparición de dichas complicaciones.

Además, estudiamos la frecuencia con que se presentaron las complicaciones post-biopsia renal y clasificamos dichas complicaciones según el grado de gravedad clínica presentada.

\section{Pacientes y métodos}

Realizamos un estudio observacional y retrospectivo en el que se incluyeron todos los pacientes trasplantados renales que precisaron BRP por indicación médica, en el periodo de tiempo comprendido entre enero de
2009 y enero 2012 en el Hospital Universitario Reina Sofía de Córdoba. Se recogió una muestra total de 92 pacientes con BRP en injerto renal, mediante la revisión de bases de datos e historias clínicas de los pacientes. Se respetó en todo momento el derecho a la confidencialidad de los pacientes y estos previamente firmaron el consentimiento informado aceptando la cesión de sus datos clínicos.

Las variables estudiadas fueron: edad, sexo, hipertensión arterial (HTA), hipotensión, proteinuria, complicaciones mayores y menores, hemoglobina anterior y posterior a la BRP, requerimiento de transfusión sanguínea tras la biopsia, número de punciones necesarias para la biopsia, creatinina y uso de desmopresina previo a la biopsia.

Como criterios de selección se incluyeron todos los pacientes trasplantados con BRP del injerto renal durante el periodo de tiempo anteriormente descrito. La indicación de biopsia renal se llevó a cabo según criterio médico y siempre se requirió la firma previa del consentimiento informado. Las indicaciones de biopsia en el trasplante renal fueron: pacientes con función retardada del injerto prolongada en el tiempo (más de 2-3 semanas) o con función renal inferior a la esperada según características del donante, pacientes con deterioro brusco de la función del injerto atribuible a enfermedad renal, pacientes con un aumento progresivo en los niveles de creatinina ( $20 \%$ respecto a creatinina basal) en un periodo de 3-6 meses, pacientes con proteinuria $>1 \mathrm{~g}$ en 24 horas y pacientes con alteraciones del sedimento sin causas urológicas aparentes.

Los criterios de exclusión utilizados fueron pacientes trasplantados sin biopsia del injerto renal debido a la ausencia de estudio de coagulación pre-biopsia, no haber suspendido el tratamiento con anticoagulantes orales, heparina, antiagregantes plaquetarios 0 antiinflamatorios no esteroideos. La presencia de crisis hipertensiva en el momento de la biopsia, alteración en la coagulación o plaquetopenia, contraindicaba la realización de BRP.

La biopsia renal percutánea se realizó mediante control ecográfico en tiempo real por el equipo de nefrología. Utilizamos un dispositivo semiautomático tipo pistola (Bard ${ }^{\circledR}$ MaxCore $^{\circledR}$ ) con aguja de 16 G, con el fin de obtener dos cilindros de tejido renal. El paciente permaneció 24 horas en la unidad con cuidados post- 
biopsia que incluyeron: medición de la tensión arterial y frecuencia cardiaca horaria, vigilancia de la diuresis y de la aparición de hematuria macroscópica, así como la presencia de dolor abdominal u otra sintomatología. Se determinó control del hematocrito a las 24 horas de la biopsia percutánea. La realización de ecografía renal y abdominal se realizó ante la presencia de hematuria macroscópica, caída del hematocrito o clínica sospechosa de sangrado.

Las variables que se estudiaron fueron: edad, sexo, hipertensión arterial, hemoglobina basal, creatinina y proteinuria. Además se analizaron las complicaciones asociadas con la biopsia renal, distinguiendo entre complicaciones mayores y menores. Por último, dividimos los pacientes en dos grupos, pacientes que presentaron alguna complicación post-biopsia, ya fuera menor o mayor, y pacientes sin complicaciones. Se compararon los dos grupos de pacientes y se analizaron las variables: edad, creatinina, hemoglobina pre-biopsia, proteinuria, descenso de hemoglobina, hipotensión, transfusión, hematuria macroscópica, número de punciones y tratamiento con desmopresina.

Las complicaciones asociadas con la biopsia renal percutánea se clasificaron como complicación mayor cuando existía necesidad de transfusión sanguínea y/o requerimiento de cirugía, nefrectomía, arteriografía, embolización o muerte. Las complicaciones menores fueron el descenso de la hemoglobina $>1 \mathrm{~g} / \mathrm{l}$ sin necesidad de transfusión sanguínea u otra técnica endovascular o quirúrgica. Para analizar las complicaciones menores, se recogieron diversos parámetros, entre ellos la hemoglobina previa y posterior a la biopsia renal.

También analizamos la presencia de hipotensión después de la biopsia, definida como caída del $20 \%$ sobre la presión arterial sistólica basal. La hipotensión se consideró complicación mayor o menor según precisara o no transfusión. Se consideró aparte ya que inicialmente nos parecía una complicación relativamente frecuente, al igual que la caída de la hemoglobina.

Los resultados de las variables cualitativas se expresan en frecuencias absolutas y en porcentajes, mientras que los resultados de las variables cuantitativas se muestran en forma de media \pm desviación estándar.

Se realizó análisis estadístico descriptivo basado en tablas de frecuencia de variables categóricas, utilizando el test de Chi-cuadrado de Pearson, para contrastar la significación de asociación entre variables cualitativas. Para comparar medias entre grupos, se utilizó la prueba de la $t$ de Student para muestras independientes.

Posteriormente, se realizó una estratificación de las tablas de contingencia con dicha variable, para analizar las diferencias entre los dos grupos. Para ello, se utilizarán la Chi-cuadrado de Pearson o el estadístico de Fisher para variables cualitativas, y el análisis de covarianza para las variables cuantitativas.

\section{Resultados}

\section{Características clínicas basales de los pacientes trasplantados renales:}

Analizamos un total de 92 biopsias de injerto renal realizados entre enero de 2009 y enero de 2012 en UGC de Nefrología en Hospital Universitario Reina Sofía. Las causas que motivaron la realización de la biopsia del injerto renal fueron el aumento de creatinina en el $89 \%$ y por proteinuria en el $11 \%$.

Las características demográficas y clínicas de los pacientes en el momento de la biopsia del injerto renal está representados en Tabla 1.

\begin{tabular}{|l|c|}
\hline & $\mathbf{N}=92$ \\
\hline Edad del paciente (años) & $47.9 \pm 13.2$ \\
\hline Sexo (\% hombres) & $70.7 \%$ \\
\hline Hemoglobina basal (g/dl) & $11.3 \pm 1.7$ \\
\hline Ser Hipertenso (\%) & $95.7 \%$ \\
\hline $\begin{array}{l}\text { Creatinina en el momento } \\
\text { de la biopsia (mg/dI) }\end{array}$ & $2.3(1.9-2.9)$ \\
\hline $\begin{array}{l}\text { Ratio Proteína/Creatinina } \\
\text { urinaria en el momento } \\
\text { de la biopsia (mg/mg) }\end{array}$ & $0.3(0.1-1.4)$ \\
\hline & \\
\hline $\begin{array}{l}\text { Las variables que se ajustan a la normalidad se expresan como } \\
\text { media } \pm \text { desviación estándar. Las variables que no se ajustan a la } \\
\text { normalidad se expresan como mediana (rango intercuartilico) }\end{array}$ \\
\hline
\end{tabular}

Tabla 1. Características demográficas y clínicas de los pacientes trasplantados en el momento de la biopsia del injerto renal 


\section{Complicaciones post-biopsia del injerto renal:}

Las complicaciones mayores se presentaron en un $2.2 \%$ de los casos, lo que corresponde a un total de 2 pacientes; como se observa en la tabla 2 . Un paciente requirió intervención quirúrgica por sangrado activo arterial, así como, transfusión de concentrados de hematíes. El otro paciente necesitó embolización selectiva por vía endovascular. Ambos pacientes conservaron su injerto renal y actualmente están funcionales.

\begin{tabular}{|c|c|}
\hline Variable & N (\%) \\
\hline Complicaciones mayores & $2(2.2 \%)$ \\
\hline Complicaciones menores & $13(14.1 \%)$ \\
\hline Caída hemoglobina & $9(9.8 \%)$ \\
\hline Presencia de hipotensión & $4(4.3 \%)$ \\
\hline \multicolumn{2}{|c|}{$\begin{array}{l}\text { Complicaciones mayores: necesidad de transfusión sanguínea, } \\
\text { requerimiento de cirugía, nefrectomía, arteriografía, embolización } \\
\text { selectiva endovascular del vaso sangrante o muerte }\end{array}$} \\
\hline \multicolumn{2}{|c|}{$\begin{array}{l}\text { Complicaciones menores: descenso de la hemoglobina }>1 \mathrm{~g} / \mathrm{l} \text {, } \\
\text { hematuria macroscópica, hipotensión, dolor en zona de punción } \\
\text { todos ellos sin necesidad de transfusión sanguínea }\end{array}$} \\
\hline \multicolumn{2}{|c|}{$\begin{array}{l}\text { Hipotensión: podrá ser considerada complicación mayor o menor } \\
\text { según precise o no transfusión. Se ha considerado aparte ya que se } \\
\text { considera una complicación relativamente frecuente }\end{array}$} \\
\hline
\end{tabular}

Tabla 2. Presencia de complicaciones mayores o menores tras la realización de la biopsia renal
Las complicaciones menores estuvieron presentes en el $14.1 \%$. Objetivamos un descenso de la hemoglobina post-biopsia renal percutánea en el $9.8 \%$, es decir en 9 pacientes, de los cuales solo precisaron transfusión dos, en el resto la caída de la hemoglobina fue leve. Recogimos como variable de estudio la hemoglobina previa y posterior a la biopsia, la media de hemoglobina pre-biopsia fue $11.3 \pm 1.7$ y post-biopsia $11.2 \pm 2.1$ $(p=0,2)$. La presencia de hipotensión tras la biopsia se objetivó en el $4.3 \%$ de las biopsias, requiriendo transfusión en un caso.

\section{Análisis de los factores implicados en la aparición de complicaciones y sintomatología post-biopsia:}

Un total de catorce pacientes ( $15.2 \%)$ presentaron alguna complicación mayor o menor tras la biopsia renal, tal como se puede apreciar en la tabla 3. Sin embargo, ningún paciente sufrió pérdida del injerto renal debido a dichas complicaciones y ningún paciente murió. Al comparar los factores clínicos en los pacientes con y sin complicaciones post-biopsia, no se encontraron diferencias significativas ni en la edad, ni en la creatinina en el momento de la biopsia, hemoglobina pre-biopsia, ratio proteína/creatinina en orina. Tampoco hubo diferencias en el número de punciones para la extracción de la muestra histológica entre los pacientes con y sin complicaciones post-biopsia.

\begin{tabular}{|c|c|c|c|}
\hline & $\begin{array}{l}\text { Pacientes con complicaciones } \\
\qquad N=14\end{array}$ & $\begin{array}{l}\text { Pacientes sin complicaciones } \\
\qquad N=78\end{array}$ & $\mathbf{p}$ \\
\hline Edad (años) & $51.86 \pm 10.4$ & $47.2 \pm 13.5$ & 0.22 \\
\hline Creatinina (mg/dl) & $2.8(2.1-5.3)$ & $2.2(1.9-2.9)$ & 0.07 \\
\hline Hemoglobina Prebiopsia ( $\mathrm{g} / \mathrm{dl}$ ) & $11.1 \pm 1.6$ & $11.4 \pm 1.7$ & 0.53 \\
\hline Proteinuria (mg/mg) & $0.4(0.15-1.6)$ & $0.29(0.15-1.4)$ & 0.70 \\
\hline Descenso hemoglobina (\%) & $57.1 \%$ & $1.3 \%$ & $<0.0001$ \\
\hline Hipotensión postbiopsia (\%) & $28.6 \%$ & $0 \%$ & $<0.0001$ \\
\hline Transfusión (\%) & $14.3 \%$ & $1.3 \%$ & 0.059 \\
\hline Hematuria macroscópica & $57.1 \%$ & $0 \%$ & $<0.0001$ \\
\hline Tratamiento con Desmopresina & $35.7 \%$ & $33.3 \%$ & 1 \\
\hline Número de punciones & $2.14 \pm 0.36$ & $2.06 \pm 0.24$ & 0.31 \\
\hline
\end{tabular}

Tabla 3. Comparación de las características clínicas de los pacientes según la presencia de complicaciones post-biopsia 
La necesidad de transfusión en los pacientes con complicaciones post-biopsia fue superior comparado a los pacientes sin complicaciones, aunque sin significación estadísticamente significativa $(p=0.059)$.

El descenso de hemoglobina en el grupo de pacientes con complicaciones fue mas elevado que en el grupo de pacientes sin complicaciones. La hipotensión postbiopsia fue mayor en pacientes con complicaciones.

\section{Discusión}

Este estudio recoge la experiencia en nuestro centro de las complicaciones tras la BRP del injerto renal. La biopsia renal es una técnica fundamental en el estudio de la patología del injerto renal. Esta técnica es necesaria para detectar la presencia de daño renal en el trasplantado cuando se está produciendo, antes del deterioro irreversible del injerto renal, para poder intervenir precozmente. La mayoría de estudios llevados a cabo sobre complicaciones tras la biopsia renal, son estudios realizados sobre biopsias en pacientes con riñones nativos y no en trasplantados renales ${ }^{2,8}$.

Estudios publicados recientemente recogen una incidencia de complicaciones mayores en la biopsia percutánea con control ecográfico menor del 5\% en riñones nativos. Igualmente en Europa y Estados Unidos las complicaciones mayores oscilan entre el 1,2 y el $6,4 \% 13,15$, semejante al porcentaje encontrado en nuestra unidad que fue del $2.2 \%$. En cuanto a las complicaciones menores, se estiman en el $6-20 \%$, siendo en nuestro análisis del $14 \%$. La incidencia de complicaciones menores en nuestro estudio fue semejante a las encontradas en la literatura en biopsias de riñones nativos ${ }^{13}$.

Nosotros creemos que nuestra baja tasa de complicaciones mayores en biopsias de trasplante renal se debe a la cumplimentación cuidadosa de nuestro protocolo de biopsia renal que optimiza la relación riesgo beneficio de esta técnica, incluso en trasplantados renales, donde los factores de riesgo para sangrado son mayores que en la población no trasplantada. Estudios previos han sugerido que la hipertensión arterial no controlada y la alteración en el estudio de coagulación incrementarían el riesgo de sangrado ${ }^{13}$.
A pesar de que el $95.7 \%$ de nuestros pacientes son hipertensos la mayoría de ellos (95\%) mostraron una presión arterial sistólica no superior a $145 \mathrm{~mm} \mathrm{mg,}$ posponiéndose la realización de la biopsia en el caso de que el paciente presentara HTA no controlada. EI estudio de coagulación siempre fue normal antes de la biopsia, suspendiendo el tratamiento con antiagregantes, antiinflamatorios no esteroideos una semana antes, así como, los tratamientos anticoagulantes. Existe controversia acerca de la correlación entre la función renal y el riesgo de complicaciones. La creatinina elevada se ha asociado con un mayor riesgo de sangrado ${ }^{15}$ y pero en otros estudios no encuentran esta asociación ${ }^{13}$. En nuestros datos no observamos relación entre niveles elevados de creatinina y una mayor incidencia de complicaciones, a pesar que el $69 \%$ de nuestros pacientes tenían una creatinina mayor de $2 \mathrm{mg} / \mathrm{dl}$ en el momento de la biopsia. Nosotros creemos que sería necesario aumentar la muestra para encontrar asociación entre la función renal y riesgo de sangrado.

Pensamos que el examen histológico de la biopsia renal sigue constituyendo a día de hoy la excelencia para la identificación diagnóstica de la patología del injerto. La obtención de material suficiente y representativo de la corteza renal, junto con la minimización de las posibles morbilidades asociadas al proceso tales como, hematuria macroscópica, fístulas arterio-venosas o hematomas perirrenales ${ }^{16}$, constituyen los principales objetivos de la biopsia diagnóstica en el paciente trasplantado. Después de analizar nuestros datos en trasplantados renales podemos afirmar que la biopsia renal percutánea realizada por médicos nefrólogos con experiencia y bajo control ecográfico en tiempo real, es un procedimiento con bajo riesgo, ya que la mayoría de las complicaciones son menores y no tienen repercusiones clínicas graves. Si bien, es necesario llevar una disciplina en el procedimiento, evitando factores de riesgo y vigilando precozmente la aparición de posibles complicaciones, como realizamos por protocolo en nuestra unidad.

Recibido: 2 Abril 2012

Revisado: 14 Abril 2012

Modificado: 10 Junio 2012

Aceptado: 20 Junio 2012 


\section{Bibliografía}

1. MedlinePlus [Base de datos en internet]. Atlanta, Georgia [actualizada el 10 de Octubre de 2011; acceso en abril de 2012]. Disponible en: http://www.nlm.nih.gov/medlineplus/spanish/ency/ article/003907.htm

2. K. Toledo, M.J. Pérez, M. Espinosa, J. Gómez, M. López, D. Redondo, R. Ortega, P. Aljama. Complicaciones asociadas a la biopsia renal percutánea. Experiencia en España 50 años después. Nefrologia 2010;30(5):539-43.

3. D. Serón, F. Anaya, R. Marcén, R. García del Moral, E. Vázquez Martul, A. Alarcón, A. Andrés, D. Burgos, L. Capdevila, M. González Molina, C. Jiménez, J. M. Morales, F. Oppenheimer, L. Pallardó y A. Sánchez Fructuoso.Recomendaciones para la indicación, obtención, procesamiento y evaluación de biopsias en el trasplante renal. Nefrología 2008; 28 (4) 385-396.

4. Peces R, De Sousa E, Peces C. La biopsia renal en situaciones especiales. Nefrología [revista en internet]* 2011 [acceso 24 de abril de 2011]; 31 (6) ). Disponible en: http://www.revistanefrologia. com/modules.php?name=articulos\&d_op=\&idarti culo $=10925 \&$ idlangart $=$ ES\&preproduccion $=$

5. García $V$, Luis $M^{a}$ I, Ruiz M. En el cincuentenario de las primeras biopsias renales percutáneas realizadas en España. Nefrología [revista en internet] 2009 [acceso 24 de abril de 2012]; 29 (1) ): [7176]. Disponible en:

http://www.revistanefrologia.com/modules.php?n ame $=$ articulos\&idarticulo $=227$

6. Rivera M. Biopsia renal ecodirigida. Nefrología [revista en internet] 2010 [acceso 27 de abril de 2012]; 30(5): [490- 492]. Disponible en: http://www.revistanefrologia.com/modules. php? name=articulos\&d_op=\&idarticulo=10537\& idlangart=ES\&preproduccion $=$

7. Francisco Rivera Hernández. Biopsia renal. NefroPlus 2009;2(1):1-8.

8. Gearoid M. McMahon, Molly E. McGovern, Vanesa Bijol, Carol B. Benson, Richard Foley, Ka- ren Munkley, Jeffrey Schnipper, Calvin Franz, Julie Lin. Development of an Outpatient Native Kidney Biopsy Service in Low-Risk Patients: A Multidisciplinary Approach. Am J Nephrol 2012;35:321-326.

9. National Kidney Federation. Kidney transplant biopsy. [actualizada el 27 de Febrero de 2011; acceso 3 de Mayo de 2012]. Disponible en: http://translate.google.es/translate?hl=es\&lan gpair=en|es\&u=http://www.kidney.org.uk/Medical-Info/kidney-disease/biopsy-transplantkidney.html

10.- Whittier W, Korbet S. El tiempo de las complicaciones en la biopsia renal percutánea. Revista de la sociedad americana de nefrología. 2003 [acceso el 27 de abril de 2012]; 15 (1): [142-147]. Disponible en: http://translate.googleusercontent. com/translate_c?hl=es\&langpair=en\%7Ces\&rurl $=$ translate.google.com\&u=http://jasn.asnjournals. org/content/15/1/142.full\&usg=ALkJrhgdRNjTn Uq02hDLsIGdhE5TIFtxnw

11.- Lefaucheur C , D Nochy, J Bariety. La biopsia renal: procedimientos, contraindicaciones, complicaciones. Pubmed. 2009 [acceso 3 de Mayo de 2012]; 5(4): [331-339]. Disponible en: http://translate.google.es/translate?hl=es\&lang pair=en\%7Ces\&u=http://www.ncbi.nlm.nih.gov/ pubmed/19345174

12. Herrero JC, Morales $E$, Carreño A, DomínguezGil B, González E, Ortiz M, Hernández E. Hemorragia grave y hematoma retroperitoneal secundario a la biopsia renal. Nephrology Dialysis Transplantation. 2001 [acceso el 7 de Mayo de 2012]; 16 (5): [1078-1079]. Disponible en: http://translate.googleusercontent.com/translate_c?hl=es\&langpair=en\%7Ces\&rurl=translat e.google.com\&u=http://ndt.oxfordjournals.org/ content/16/5/1078.full\&usg=ALkJrhi-WempVVg5oI8rJGleKvmBBW5E-A

13. Anno C, Strippoli G, Unrnesano L, Onifati C, Ampobasso N, Esualdo L, Chena F. Los predictores de complicaciones hemorrágicas en la biopsia percutánea guiada por ecografía renal. Kidney international. 2004 [acceso el 7 de Mayo de 2012]; 66: [1570-1577]. Disponible en: http:// 
translate.google.es/translate?hl=es\&langpair=e n\%7Ces\&u=http://www.nature.com/ki/journal/ v66/n4/full/4494783a.html

14. Kidney Biopsy. Mayo Clinic. 2010 [acceso el 13 de Mayo de 2012]. Disponible en: http://www. mayoclinic.com/health/kidney-biopsy/MY01223/ DSECTION=risks

15. Mackinnon B, Fraser E, Simpson K, Fox J, Geddes C. Is it necessary to stop antiplatelet agents be- fore a native renal biopsy? Nephrol Dial Transplant 2000;23:3566-70.

16. Vidhun J, Masciandro J, Varich L, Salvatierra 0, Jr., Sarwal M. Safety and risk stratification of percutaneous biopsies of adult-sized renal allografts in infant and older pediatric recipients. Transplantation 2003; 76 (3): 552-7. 